\title{
Well-Posedness for a Modified Bidomain Model Describing Bioelectric Activity in Damaged Heart Tissues
}

\author{
M. Amar@, D. Andreucci and C. Timofte
}

\begin{abstract}
We prove the existence and the uniqueness of a solution for a modified bidomain model, describing the electrical behaviour of the cardiac tissue in pathological situations. The leading idea is to reduce the problem to an abstract parabolic setting, which requires to introduce several auxiliary differential systems and a non-standard bilinear form. The main difficulties are due to the degeneracy of the bidomain system and to its non-standard coupling with a diffusion equation, accounting for the presence of the pathological zone in the heart tissue.
\end{abstract}

Mathematics Subject Classification. 35K90, 35A01, 35K20, 35Q92.

Keywords. Existence, uniqueness, abstract parabolic equations, bidomain model, imperfect transmission conditions.

\section{Introduction}

In this paper, we are interested in studying a modified version of the famous bidomain model (see, e.g., $[10,19,20]$ and the references therein; see, also, the references quoted in [11, Introduction]), which is one the most well-known mathematical models in cardiac electrophysiology. This is a topic of major interest in biomedical research.

In the classical bidomain model, at a macroscopic scale, the electric activity of the heart is governed by a system of two degenerate reactiondiffusion partial differential equations for the averaged intra-cellular and, respectively, extra-cellular electric potentials, along with the transmembrane potential, coupled in a nonlinear manner to ordinary differential equations describing the dynamics of the ion channels. The well-posedness of the bidomain model has been studied, for different nonlinear ionic models and using different techniques, by several authors (see, for instance, $[3,8,11,14,15,18$, $21,23,24])$.

The bidomain model is suitable for describing the propagation of the action potential in a perfectly healthy cardiac tissue, but it is no longer valid 
(even if one tries to ad-hoc modify some of its relevant modeling parameters) in pathological situations. Models, taking into account the presence in the cardiac tissue of damaged zones, called diffusive inclusions and assumed to be passive electrical conductors, were proposed in $[6,12-14]$.

From a mathematical point of view, such models consist in a bidomain system coupled with a diffusion equation. More precisely, one has a degenerate reaction-diffusion system of partial differential equations modeling the intra-cellular and, respectively, the extra-cellular electric potentials of the healthy cardiac tissue, coupled with an elliptic equation for the passive regions and with an ordinary differential equation describing the cellular membrane dynamics.

We point out that in all the above mentioned papers, a perfect electrical coupling between the healthy part of the heart and the damaged tissue was assumed. More general conditions for the heart-torso coupling were proposed in [7] and investigated through numerical simulations in $[4,5,26]$, to take into account the possible capacitive and resistive effects of the pericardium. However, up to our knowledge, there are no rigorous proofs in the literature covering this setting. We investigate these more general conditions in the context of the bidomain model with diffusive inclusions, where the appropriate interface behaviour, up to our knowledge, is still not well understood.

The goal of the present paper is to study the well-posedness of such a modified bidomain model. We include the structural defects of the heart tissue in this model by coupling a standard bidomain system in the healthy zone with a diffusion equation posed in the damaged part of the heart, through non-standard conditions (see Eqs. (2.15)-(2.20)). More precisely, for the intracellular potential, we assume no flux condition on the interface between the two zones (see (2.18)), while the extra-cellular potential is coupled with the electrical potential of the damaged zone through imperfect transmission conditions, involving the resistive and the capacitive properties of the interface (see (2.19) and (2.20)).

To describe the dynamic of the membrane, one can use a physiological ionic model or a phenomenological one (see, for instance, [11]). In this paper, the dynamic of the gating variable modeling the ionic transport through the cell membrane is described with the aid of a Hodgkin-Huxley type formalism (see (2.10)-(2.13)). Our analysis covers also the modified Mitchell-Schaeffer formalism proposed in [13] (see Remark 2.3).

We point out again that our mathematical model generalizes the modified bidomain model with diffusive inclusions and perfect transmission conditions considered in $[13,14]$, the original model being recovered by suitably rearranging the parameters appearing in Eq. (2.20). We believe that further numerical simulations have to be carried out to validate the relevance of such transmission conditions also from the point of view of possible biological applications.

The mathematical problem we address here is rather non-standard and, up to our knowledge, the proof of its well-posedness is new in the literature and generates difficulties due to the degeneracy of the bidomain system and to its special coupling with the diffusion equation. 

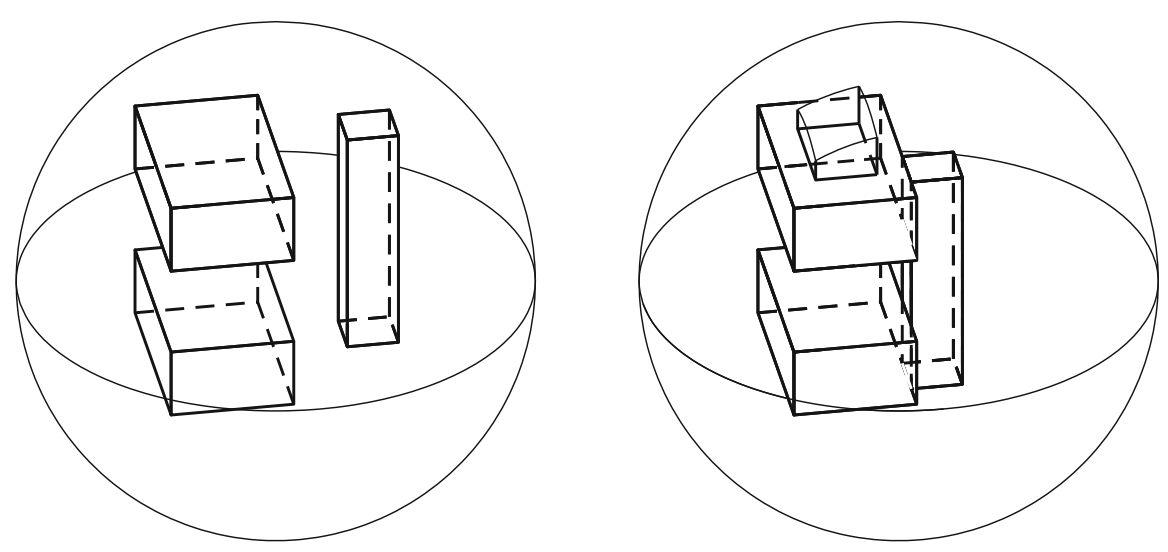

Figure 1. On the left: the connected/disconnected case. On the right: the connected/connected case

Our main result is contained in Theorems 3.4 and 3.6, where the leading idea is to reduce the problem to an abstract parabolic setting (see $[9,22]$ ). This requires to introduce several auxiliary differential systems and a nonstandard bilinear form (see Proposition 3.3).

The problem proposed here can be seen as a mesoscopic model which will be analyzed in the homogenization limit in a forthcoming article (see $[2])$.

The paper is organized as follows: in Sect. 2, we introduce the mathematical description of our modified bidomain model, together with its geometrical and functional setting. In Sect. 3, we state and prove our main result.

\section{The Model}

\subsection{Geometrical Setting}

Let $N \geq 3$. Let $\Omega$ be an open connected bounded subset of $\mathbb{R}^{N}$; we assume that $\partial \Omega$ is of class $\mathcal{C}^{\infty}$, though this assumption can be weakened. Moreover, for $T>0$, we set $\Omega_{T}=\Omega \times(0, T)$. We assume that $\Omega=\Omega^{D} \cup \Omega^{B} \cup \Gamma$, where $\Omega^{D}$ and $\Omega^{B}$ are two disjoint open subsets of $\Omega$ and $\Gamma=\partial \Omega^{D} \cap \Omega=$ $\partial \Omega^{B} \cap \Omega$. The domain $\Omega$ is occupied by the cardiac tissue, $\Omega^{B}$ represents the healthy part of the heart tissue, modeled with the aid of a standard bidomain system, $\Omega^{D}$ represents the diffusive region, accounting for the damaged part of the heart, and $\Gamma$ is the common boundary of these two regions, assumed to be Lipschitz. From a geometrical point of view, we assume that $\Omega^{B}$ is connected, while $\Omega^{D}$ might be connected or disconnected. Indeed, we will consider two different cases: in the first one (to which we will refer as the connected/disconnected case, see Fig. 1 on the left), we will assume $\Omega^{D} \subset \subset \Omega$ and $\Omega^{D}$ is made by a finite number of connected components. In this case, $\Gamma=\partial \Omega^{D}$ and $\partial \Omega^{B} \cap \partial \Omega \neq \emptyset$.

In the second case (to which we will refer as the connected/connected case, see Fig. 1 on the right), we will assume that both $\Omega^{D}$ and $\Omega^{B}$ are 
connected, with $\partial \Omega^{B} \cap \partial \Omega \neq \emptyset$ and $\partial \Omega^{D} \cap \partial \Omega \neq \emptyset$. Finally, let $\nu$ denote the normal unit vector to $\Gamma$ pointing into $\Omega^{B}$.

In the following, by $\gamma$, we shall denote a strictly positive constant, which may depend on the geometry and on the other parameters of the problem; $\gamma$ may vary from line to line.

\subsection{Functional Spaces}

Let us introduce the following functional spaces:

$H_{\text {null }}^{1}\left(\Omega^{B}\right):=\left\{w \in H^{1}\left(\Omega^{B}\right): w=0\right.$ on $\partial \Omega^{B} \cap \partial \Omega$, in the sense of traces $\}$; $H_{\text {null }}^{1}\left(\Omega^{D}\right):=\left\{w \in H^{1}\left(\Omega^{D}\right): w=0\right.$ on $\partial \Omega^{D} \cap \partial \Omega$, in the sense of traces $\}$; $H_{0}^{1 / 2}(\Gamma, \Omega):=\left\{r \in H^{1 / 2}(\Gamma): r=\widetilde{r}_{\left.\right|_{\Gamma}}\right.$, with $\left.\widetilde{r} \in H_{0}^{1}(\Omega)\right\}$.

Notice that $H_{0}^{1 / 2}(\Gamma, \Omega)$ is a Hilbert space and, in the connected/disconnected case, $H_{0}^{1 / 2}(\Gamma, \Omega)=H^{1 / 2}(\Gamma)$ and $H_{\text {null }}^{1}\left(\Omega^{D}\right)=H^{1}\left(\Omega^{D}\right)$.

We also set

$$
W:=H_{\text {null }}^{1}\left(\Omega^{B}\right) \times H_{0}^{1 / 2}(\Gamma, \Omega) ; \quad H:=L^{2}\left(\Omega^{B}\right) \times L^{2}(\Gamma),
$$

where $H$ is endowed with the scalar product

$$
((w, r),(\bar{w}, s))_{H}:=\int_{\Omega^{B}} w \bar{w} \mathrm{~d} x+\alpha \int_{\Gamma} r s \mathrm{~d} \sigma ;
$$

here, $\alpha>0$ will be the constant appearing later in (2.20) and $W$ is endowed with the scalar product

$$
((w, r),(\bar{w}, s))_{W}:=\int_{\Omega^{B}} \nabla w \cdot \nabla \bar{w} \mathrm{~d} x+(r, s)_{1 / 2} .
$$

We denote by $(\cdot, \cdot)_{1 / 2}$ the standard scalar product on $H^{1 / 2}(\Gamma)$.

Moreover, we define the space

$$
\mathcal{X}_{0}^{1}(\Omega):=\left\{\mathcal{W}: \Omega \rightarrow \mathbb{R}: \mathcal{W}_{\left.\right|_{\Omega^{B}}} \in H_{\text {null }}^{1}\left(\Omega^{B}\right), \mathcal{W}_{\left.\right|_{\Omega^{D}}} \in H_{\text {null }}^{1}\left(\Omega^{D}\right)\right\}
$$

endowed with the norm

$$
\|\mathcal{W}\|_{\mathcal{X}_{0}^{1}(\Omega)}^{2}:=\|\nabla \mathcal{W}\|_{L^{2}\left(\Omega^{B}\right)}^{2}+\|\mathcal{W}\|_{H^{1}\left(\Omega^{D}\right)}^{2} .
$$

We recall that $\partial \Omega^{B} \cap \partial \Omega$ is always non-empty, while $\partial \Omega^{D}$ can intersect or not the boundary of $\Omega$, depending on the geometry. For $\mathcal{W} \in \mathcal{X}_{0}^{1}(\Omega)$, we have the following Poincaré inequality (see [1, Proposition 2]):

$$
\|\mathcal{W}\|_{L^{2}(\Omega)}^{2} \leq \gamma\left(\|\nabla \mathcal{W}\|_{L^{2}\left(\Omega^{B}\right)}^{2}+\|\nabla \mathcal{W}\|_{L^{2}\left(\Omega^{D}\right)}^{2}+\|[\mathcal{W}]\|_{L^{2}(\Gamma)}^{2}\right)
$$

where $[\mathcal{W}]=\mathcal{W}_{\left.\right|_{\Omega^{B}}}-\mathcal{W}_{\left.\right|_{\Omega^{D}}}$ and the last term is not necessary in the connected/connected case. Therefore, an equivalent norm on $\mathcal{X}_{0}^{1}(\Omega)$ is given by

$$
\|\mathcal{W}\|_{\mathcal{X}_{0}^{1}(\Omega)}^{2} \sim\|\nabla \mathcal{W}\|_{L^{2}(\Omega)}^{2}+\|[\mathcal{W}]\|_{L^{2}(\Gamma)}^{2}
$$

again, the last term can be dropped in the connected/connected case. 


\subsection{Position of the Problem}

Let $\alpha, \beta$ be strictly positive constants and $\sigma_{1}^{B}, \sigma_{2}^{B}, \sigma^{D}$ be measurable functions, such that $\gamma_{0} \leq \sigma_{1}^{B}(x), \sigma_{2}^{B}(x), \sigma^{D}(x) \leq \widetilde{\gamma}_{0}$, a.e. in $\Omega$, for suitable strictly positive constants $\gamma_{0}, \widetilde{\gamma}_{0}$. The assumption that $\sigma_{1}^{B}, \sigma_{2}^{B}, \sigma^{D}$ are scalar functions is used only in Sect. 3. Removing this assumption is not trivial. If we want to consider general bounded and symmetric matrices satisfying

$$
\begin{array}{ll}
\gamma_{0}|\zeta|^{2} \leq \sigma_{1}^{B}(x) \zeta \cdot \zeta \leq \widetilde{\gamma}_{0}|\zeta|^{2}, & \text { for every } \zeta \in \mathbb{R}^{N} \text { and a.e. } x \in \Omega^{B} ; \\
\gamma_{0}|\zeta|^{2} \leq \sigma_{2}^{B}(x) \zeta \cdot \zeta \leq \widetilde{\gamma}_{0}|\zeta|^{2}, & \text { for every } \zeta \in \mathbb{R}^{N} \text { and a.e. } x \in \Omega^{B} ; \\
\gamma_{0}|\zeta|^{2} \leq \sigma^{D}(x) \zeta \cdot \zeta \leq \widetilde{\gamma}_{0}|\zeta|^{2}, & \text { for every } \zeta \in \mathbb{R}^{N} \text { and a.e. } x \in \Omega^{D},
\end{array}
$$

we have to require some other structural hypotheses as in [8, Lemma 1] and [17, Formula (1)] (see, also, $[3,4,14]$ ).

Let us consider a locally Lipschitz continuous function $g: \mathbb{R}^{2} \rightarrow \mathbb{R}$, such that $g(p, 1) \geq 0$ and $g(p, 0) \leq 0$. The example we have in mind here is a function of the form

$$
g(p, q)=a(p)(q-1)+b(p) q,
$$

where $a, b: \mathbb{R} \rightarrow \mathbb{R}$ are positive, bounded, and Lipschitz functions. Notice that the form of $g$ in (2.10) is classical in this framework (see, for instance, [24]) and that $g$ is Lipschitz continuous with respect to $p$ and affine with respect to $q$. Let $I_{\text {ion }}: \mathbb{R}^{2} \rightarrow \mathbb{R}$ be given by

$$
I_{\text {ion }}(p, q)=h_{1}(p)+h_{2}(p) q,
$$

where $h_{1}, h_{2}$ are Lipschitz continuous functions and $h_{2}$ is bounded. Let $\mathrm{w}_{o} \in$ $L^{\infty}\left(\Omega^{B}\right)$, with $0 \leq \mathrm{w}_{o}(x) \leq 1$ a.e. in $\Omega^{B}$, and $p \in L^{2}\left(\Omega_{T}^{B}\right)$. Consider the gating equation

$$
\begin{gathered}
\partial_{t} \widetilde{w}_{p}+g\left(p, \widetilde{w}_{p}\right)=0, \quad \text { in } \quad \Omega_{T}^{B} ; \\
\widetilde{w}_{p}(x, 0)=\mathrm{W}_{o}(x), \quad \text { in } \quad \Omega^{B} .
\end{gathered}
$$

Notice that, by classical results, the previous problem admits a unique solution $\widetilde{w}_{p} \in H^{1}\left(0, T ; L^{\infty}\left(\Omega^{B}\right)\right)$ and, from our assumptions, $0 \leq \widetilde{w}_{p}(x, t) \leq 1$ a.e. in $\Omega_{T}^{B}$, since $0 \leq \mathrm{W}_{o}(x) \leq 1$ a.e. in $\Omega^{B}$. This is a standard result for ODEs, taking into account that the spatial variable plays here only the role of a parameter (for similar results, see, for instance, $[11,13,16]$ ).

Moreover, from the previous assumptions, we can prove that there exists a strictly positive constant $\gamma_{I}$, such that

$$
\left\|I_{\text {ion }}\left(p_{1}, \widetilde{w}_{p_{1}}\right)-I_{\text {ion }}\left(p_{2}, \widetilde{w}_{p_{2}}\right)\right\|_{L^{2}\left(\Omega_{T}^{B}\right)} \leq \gamma_{I}\left\|p_{1}-p_{2}\right\|_{L^{2}\left(\Omega_{T}^{B}\right)},
$$

due to the Lipschitz dependence of $\widetilde{w}_{p}$ on $p$ and to the bound $0 \leq \widetilde{w}_{p}(x, t) \leq 1$ a.e. in $\Omega_{T}^{B}$.

We give here a complete formulation of the problem we shall address in this paper. The operators div and $\nabla$ act only with respect to the space variable $x$. 
Let $f_{1}, f_{2} \in H^{1}\left(\Omega_{T}^{B}\right), \bar{v}_{0} \in L^{2}\left(\Omega^{B}\right), s_{0} \in L^{2}(\Gamma)$ and consider the problem for $u_{1}^{B}, u_{2}^{B} \in L^{2}\left(0, T ; H_{\text {null }}^{1}\left(\Omega^{B}\right)\right), u^{D} \in L^{2}\left(0, T ; H_{\text {null }}^{1}\left(\Omega^{D}\right)\right)$ and $\widetilde{w} \in H^{1}\left(0, T ; L^{\infty}\left(\Omega^{B}\right)\right)$ given by

$$
\begin{gathered}
\frac{\partial}{\partial t}\left(u_{1}^{B}-u_{2}^{B}\right)-\operatorname{div}\left(\sigma_{1}^{B} \nabla u_{1}^{B}\right)+I_{\text {ion }}\left(u_{1}^{B}-u_{2}^{B}, \widetilde{w}\right)=f_{1}, \quad \text { in } \quad \Omega_{T}^{B} \\
\frac{\partial}{\partial t}\left(u_{1}^{B}-u_{2}^{B}\right)+\operatorname{div}\left(\sigma_{2}^{B} \nabla u_{2}^{B}\right)+I_{\text {ion }}\left(u_{1}^{B}-u_{2}^{B}, \widetilde{w}\right)=f_{2}, \quad \text { in } \quad \Omega_{T}^{B} ; \\
-\operatorname{div}\left(\sigma^{D} \nabla u^{D}\right)=0, \quad \text { in } \quad \Omega_{T}^{D} ; \\
\sigma_{1}^{B} \nabla u_{1}^{B} \cdot \nu=0, \quad \text { on } \quad \Gamma_{T} ; \\
\sigma_{2}^{B} \nabla u_{2}^{B} \cdot \nu=\sigma^{D} \nabla u^{D} \cdot \nu, \quad \text { on } \quad \Gamma_{T} ; \\
\alpha \frac{\partial}{\partial t}\left(u_{2}^{B}-u^{D}\right)+\beta\left(u_{2}^{B}-u^{D}\right)=\sigma_{2}^{B} \nabla u_{2}^{B} \cdot \nu, \quad \text { on } \quad \Gamma_{T} ; \\
u_{1}^{B}(x, t), u_{2}^{B}(x, t), u^{D}(x, t)=0, \quad \text { on } \partial \Omega \times(0, T) ; \\
u_{1}^{B}(x, 0)-u_{2}^{B}(x, 0)=\bar{v}_{0}(x), \quad \text { in } \quad \Omega^{B} ; \\
u_{2}^{B}(x, 0)-u^{D}(x, 0)=s_{0}(x), \quad \text { on } \quad \Gamma
\end{gathered}
$$

where $\widetilde{w}$ is the solution of the gating Eqs. (2.12) and (2.13), with $p=u_{1}^{B}-u_{2}^{B}$.

Remark 2.1. (Biological interpretation) In the previous system of equations, the coefficients $\sigma_{1}^{B}, \sigma_{2}^{B}$ and $\sigma^{D}$ are the conductivities of the two healthy phases and of the damaged one, respectively, while $\alpha$ and $\beta$ are given parameters related to the capacitive and the resistive behaviour of the interface $\Gamma$. The functions $f_{1}$ and $f_{2}$, appearing in (2.15) and (2.16), respectively, represent the internal and the external current stimulus. The solutions $u_{1}^{B}$ and $u_{2}^{B}$ are the intra- and the extra-cellular potentials of the healthy zone, while $u^{D}$ is the electrical potential of the damaged zone. The function $u_{2}^{B}-u_{1}^{B}$ is the so-called transmembrane potential. Finally, the variable $\widetilde{w}$, called the gating variable, describes the ionic transport through the cell membrane. The terms $g$ and $I_{\text {ion }}$ are nonlinear functions, modeling the membrane ionic currents. For simplicity, we consider only one gating variable, but our results hold true also for the case in which the gating variable is vector valued.

Remark 2.2. In Eq. (2.18), we are assuming that the intra-cellular potential satisfies a no flux condition on the interface $\Gamma_{T}$ between the two zones $\Omega^{B}$ and $\Omega^{D}$, while Eqs. (2.19) and (2.20) state the imperfect transmission conditions coupling the extra-cellular potential with the electrical potential of the damaged zone $\Omega^{D}$. Such conditions involve the capacitive and the resistive properties of the interface, represented by the constants $\alpha$ and $\beta$, respectively.

Remark 2.3. Different examples of functions $I_{\text {ion }}$ and $g$ are considered in the literature. We consider here a Hodgkin-Huxley type model (see (2.10)$(2.11))$, as in $[2,11,24]$. However, we point out that the results obtained in this paper are also valid for a regularized version of the Mitchell-Schaeffer model proposed in [13] (see, also, $[12,14,18]$ ). 
By standard approximation procedure, multiplying (2.15) by $u_{1}^{B},(2.16)$ by $u_{2}^{B},(2.17)$ by $u^{D}$, subtracting (2.16) from (2.15), adding (2.17), integrating by parts, using (2.18)-(2.23), (2.11), (2.14) and moving the integral containing $I_{\text {ion }}$ to the right-hand side, we get

$$
\begin{aligned}
\frac{1}{2} \int_{\Omega^{B}} & \left(u_{1}^{B}-u_{2}^{B}\right)^{2}(x, T) \mathrm{d} x+\int_{\Omega_{T}^{B}} \sigma_{1}^{B}\left|\nabla u_{1}^{B}\right|^{2} \mathrm{~d} x \mathrm{~d} t+\int_{\Omega_{T}^{B}} \sigma_{2}^{B}\left|\nabla u_{2}^{B}\right|^{2} \mathrm{~d} x \mathrm{~d} t \\
& +\int_{\Omega_{T}^{D}} \sigma^{D}\left|\nabla u^{D}\right|^{2} \mathrm{~d} x \mathrm{~d} t+\frac{\alpha}{2} \int_{\Gamma}\left(u_{2}^{B}-u^{D}\right)^{2}(x, T) \mathrm{d} \sigma(x) \\
& +\beta \int_{\Gamma_{T}}\left(u_{2}^{B}-u^{D}\right)^{2}(x, t) \mathrm{d} \sigma(x) \mathrm{d} t \\
= & \int_{\Omega_{T}^{B}}\left(f_{1} u_{1}^{B}-f_{2} u_{2}^{B}\right) \mathrm{d} x \mathrm{~d} t+\frac{1}{2} \int_{\Omega^{B}} \bar{v}_{0}^{2}(x) \mathrm{d} x+\frac{\alpha}{2} \int_{\Gamma} s_{0}^{2}(x) \mathrm{d} \sigma(x) \\
& -\int_{\Omega_{T}^{B}} I_{\text {ion }}\left(u_{1}^{B}-u_{2}^{B}, \widetilde{w}\right)\left(u_{1}^{B}-u_{2}^{B}\right) \mathrm{d} x \mathrm{~d} t \\
\leq & \int_{\Omega_{T}^{B}}\left(f_{1} u_{1}^{B}-f_{2} u_{2}^{B}\right) \mathrm{d} x \mathrm{~d} t+\gamma\left(\left\|\bar{v}_{0}\right\|_{L^{2}\left(\Omega^{B}\right)}^{2}+\left\|s_{0}\right\|_{L^{2}(\Gamma)}^{2}\right) \\
& -\int_{\Omega_{T}^{B}}\left(I_{\text {ion }}\left(u_{1}^{B}-u_{2}^{B}, \widetilde{w}\right)-I_{\text {ion }}\left(0, w_{0}\right)\right)\left(u_{1}^{B}-u_{2}^{B}\right) \mathrm{d} x \mathrm{~d} t \\
& -\int_{\Omega_{T}^{B}} I_{\text {ion }}\left(0, w_{0}\right)\left(u_{1}^{B}-u_{2}^{B}\right) \mathrm{d} x \mathrm{~d} t \\
\leq & \gamma\left(\left\|f_{1}\right\|_{L^{2}\left(\Omega_{T}^{B}\right)}^{2}+\left\|f_{2}\right\|_{L^{2}\left(\Omega_{T}^{B}\right)}^{2}+\left\|\bar{v}_{0}\right\|_{L^{2}\left(\Omega^{B}\right)}^{2}+\left\|s_{0}\right\|_{L^{2}(\Gamma)}^{2}+1\right) \\
& \left.+\frac{\delta}{2}\left(\left\|\nabla u_{1}^{B}\right\|_{L^{2}\left(\Omega_{T}^{B}\right)}^{2}+\left\|\nabla u_{2}^{B}\right\|_{L^{2}\left(\Omega_{T}^{B}\right)}^{2}\right)+\gamma\left\|u_{1}^{B}-u_{2}^{B}\right\|_{L^{2}(\Omega)}^{2}\right),
\end{aligned}
$$

where $\gamma$ and $\delta$ are positive constants, $\delta$ can be chosen smaller than $\min \left(\sigma_{1}^{B}, \sigma_{2}^{B}\right)$, and we have also applied Poincaré inequality to $u_{1}^{B}$ and $u_{2}^{B}$. By absorbing into the left-hand side the first two terms in the last line of (2.24) and using Gronwall inequality, from the previous estimate, we obtain

$$
\begin{aligned}
& \sup _{t \in(0, T)} \int_{\Omega^{B}}\left(u_{1}^{B}-u_{2}^{B}\right)^{2}(x, t) \mathrm{d} x+\int_{\Omega_{T}^{B}}\left|\nabla u_{1}^{B}\right|^{2} \mathrm{~d} x \mathrm{~d} t \\
& \quad+\int_{\Omega_{T}^{B}}\left|\nabla u_{2}^{B}\right|^{2} \mathrm{~d} x \mathrm{~d} t+\int_{\Omega_{T}^{D}}\left|\nabla u^{D}\right|^{2} \mathrm{~d} x \mathrm{~d} t \\
& \quad+\sup _{t \in(0, T)} \int_{\Gamma}\left(u_{2}^{B}-u^{D}\right)^{2}(x, t) \mathrm{d} \sigma(x)+\int_{\Gamma_{T}}\left(u_{2}^{B}-u^{D}\right)^{2}(x, t) \mathrm{d} \sigma(x) \mathrm{d} t \\
& \leq \gamma\left(\left\|f_{1}\right\|_{L^{2}\left(\Omega_{T}^{B}\right)}^{2}+\left\|f_{2}\right\|_{L^{2}\left(\Omega_{T}^{B}\right)}^{2}+\left\|\bar{v}_{0}\right\|_{L^{2}\left(\Omega^{B}\right)}^{2}+\left\|s_{0}\right\|_{L^{2}(\Gamma)}^{2}+1\right) .
\end{aligned}
$$

Proposition 2.4. Under the assumptions stated above, problem (2.15)-(2.23) and (2.12)-(2.13) admits at most one solution.

Proof. Assume that $\left(u_{1}^{B}, u_{2}^{B}, u^{D}, \widetilde{w}\right)$ and $\left(\bar{u}_{1}^{B}, \bar{u}_{2}^{B}, \bar{u}^{D}, \overline{\widetilde{w}}\right)$ are two different solutions of problem (2.15)-(2.22), with $\overline{\widetilde{w}}$ being the solution of (2.12)-(2.13), 
corresponding to $p=\bar{u}_{1}^{B}-\bar{u}_{2}^{B}$. Setting $v_{1}^{B}:=u_{1}^{B}-\bar{u}_{1}^{B}, v_{2}^{B}:=u_{2}^{B}-\bar{u}_{2}^{B}$ and $v^{D}:=u^{D}-\bar{u}^{D}$, we obtain that $v_{1}^{B}, v_{2}^{B}, v^{D}, \widetilde{w}$ and $\widetilde{\widetilde{w}}$ solve the system

$$
\begin{gathered}
\frac{\partial}{\partial t}\left(v_{1}^{B}-v_{2}^{B}\right)-\operatorname{div}\left(\sigma_{1}^{B} \nabla v_{1}^{B}\right)+I_{\text {ion }}\left(u_{1}^{B}-u_{2}^{B}, \widetilde{w}\right)-I_{\text {ion }}\left(\bar{u}_{1}^{B}-\bar{u}_{2}^{B}, \overline{\widetilde{w}}\right)=0, \quad \text { in } \Omega_{T}^{B} ; \\
\frac{\partial}{\partial t}\left(v_{1}^{B}-v_{2}^{B}\right)+\operatorname{div}\left(\sigma_{2}^{B} \nabla v_{2}^{B}\right)+I_{\text {ion }}\left(u_{1}^{B}-u_{2}^{B}, \widetilde{w}\right)-I_{\text {ion }}\left(\bar{u}_{1}^{B}-\bar{u}_{2}^{B}, \overline{\widetilde{w}}\right)=0, \quad \text { in } \Omega_{T}^{D} ; \\
-\operatorname{div}\left(\sigma^{D} \nabla v^{D}\right)=0, \quad \text { in } \Omega_{T}^{D} ; \\
\sigma_{1}^{B} \nabla v_{1}^{B} \cdot \nu=0, \quad \text { on } \Gamma_{T} ; \\
\sigma_{2}^{B} \nabla v_{2}^{B} \cdot \nu=\sigma^{D} \nabla v^{D} \cdot \nu, \quad \text { on } \Gamma_{T} ; \\
\alpha \frac{\partial}{\partial t}\left(v_{2}^{B}-v^{D}\right)+\beta\left(v_{2}^{B}-v^{D}\right)=\sigma_{2}^{B} \nabla v_{2}^{B} \cdot \nu, \quad \text { on } \Gamma_{T} ; \\
v_{1}^{B}(x, t), v_{2}^{B}(x, t), v^{D}(x, t)=0, \quad \text { on } \partial \Omega \times(0, T) ; \\
v_{1}^{B}(x, 0)-v_{2}^{B}(x, 0)=0, \quad \text { in } \Omega^{B} ; \\
v_{2}^{B}(x, 0)-v^{D}(x, 0)=0, \quad \text { on } \Gamma .
\end{gathered}
$$

Reasoning in a similar way as done for (2.24), i.e., by multiplying the first equation by $v_{1}^{B}$, the second one by $v_{2}^{B}$, the third one by $v^{D}$, subtracting the second equation from the first one, adding the third one, integrating by parts, using the remaining equation of the previous system moving the integral containing $I_{\text {ion }}$ to the right-hand side and using Hölder inequality, we get

$$
\begin{aligned}
& \sup _{t \in(0, T)} \int_{\Omega^{B}}\left(v_{1}^{B}-v_{2}^{B}\right)^{2}(x, t) \mathrm{d} x+\int_{\Omega_{T}^{B}}\left|\nabla v_{1}^{B}\right|^{2} \mathrm{~d} x \mathrm{~d} t \\
& \quad+\int_{\Omega_{T}^{B}}\left|\nabla v_{2}^{B}\right|^{2} \mathrm{~d} x \mathrm{~d} t+\int_{\Omega_{T}^{D}}\left|\nabla v^{D}\right|^{2} \mathrm{~d} x \mathrm{~d} t \\
& \quad+\sup _{t \in(0, T)} \int_{\Gamma}\left(v_{2}^{B}-v^{D}\right)^{2}(x, t) \mathrm{d} \sigma(x)+\int_{\Gamma_{T}}\left(v_{2}^{B}-v^{D}\right)^{2}(x, t) \mathrm{d} \sigma(x) \mathrm{d} t \\
& \leq \| I_{\text {ion }}\left(u_{1}^{B}-u_{2}^{B}, \widetilde{w}\right)-I_{\text {ion }}\left(\bar{u}_{1}^{B}-\bar{u}_{2}^{B}, \overline{\widetilde{w})} \|_{L^{2}\left(\Omega_{T}^{B}\right)}\right. \\
& \quad \cdot\left\|v_{1}^{B}-v_{2}^{B}\right\|_{L^{2}\left(\Omega_{T}^{B}\right) \leq \gamma\left\|v_{1}^{B}-v_{2}^{B}\right\|_{L^{2}\left(\Omega_{T}^{B}\right)}^{2},}
\end{aligned}
$$

where, in the last inequality, we used (2.14). We can conclude using Gronwall inequality.

Notice that, by setting $V=u_{1}^{B}-u_{2}^{B}, U=u_{2}^{B}$ a.e. in $\Omega^{B}, U=u^{D}$ a.e. in $\Omega^{D}$, and denoting by $[\cdot]$ the jump across $\Gamma$ of the quantity in the square brackets, i.e., $[U]=u_{2}^{B}-u^{D}$ and $[\sigma \nabla U \cdot \nu]=\left(\sigma_{2}^{B} \nabla u_{2}^{B}-\sigma^{D} \nabla u^{D}\right) \cdot \nu$, the system (2.15)-(2.23) can be written in the more convenient form

$$
\begin{gathered}
\frac{\partial V}{\partial t}-\operatorname{div}\left(\sigma_{1}^{B} \nabla V\right)+I_{\text {ion }}(V, \widetilde{w})=f_{1}+\operatorname{div}\left(\sigma_{1}^{B} \nabla U\right), \text { in } \Omega_{T}^{B} \\
-\operatorname{div}\left(\left(\sigma_{1}^{B}+\sigma_{2}^{B}\right) \nabla U\right)=f_{1}-f_{2}+\operatorname{div}\left(\sigma_{1}^{B} \nabla V\right), \text { in } \Omega_{T}^{B} \\
-\operatorname{div}\left(\sigma^{D} \nabla U\right)=0, \text { in } \Omega_{T}^{D} ; \\
\sigma_{1}^{B} \nabla(V+U) \cdot \nu=0, \text { on } \Gamma_{T} ;
\end{gathered}
$$




$$
\begin{gathered}
{[\sigma \nabla U \cdot \nu]=0, \quad \text { on } \quad \Gamma_{T} ;} \\
\alpha \frac{\partial}{\partial t}[U]+\beta[U]=\sigma_{2}^{B} \nabla U \cdot \nu, \quad \text { on } \quad \Gamma_{T} ; \\
V, U=0, \quad \text { on } \partial \Omega \times(0, T) \\
V(x, 0)=\bar{v}_{0}(x), \quad \text { in } \quad \Omega^{B} ; \\
{[U](x, 0)=s_{0}(x), \quad \text { on } \quad \Gamma}
\end{gathered}
$$

complemented with the gating problem (2.12)-(2.13), where again $u_{1}^{B}-u_{2}^{B}$ is replaced by $V$. Clearly, $V \in L^{2}\left(0, T ; H_{\text {null }}^{1}\left(\Omega^{B}\right)\right)$ and $U \in L^{2}\left(0, T ; \mathcal{X}_{0}^{1}(\Omega)\right)$. We recall that, by $(2.14)$, the composed function $I_{\text {ion }}(V, \widetilde{w})$ is also a Lipschitz function with respect to $V$.

The weak formulation of the previous problem is given by

$$
\begin{aligned}
& -\int_{\Omega_{T}^{B}} V \partial_{t} \varphi_{B} \mathrm{~d} x \mathrm{~d} t+\int_{\Omega_{T}^{B}} \sigma_{1}^{B} \nabla V \cdot \nabla \varphi_{B} \mathrm{~d} x \mathrm{~d} t \\
& \quad+\int_{\Omega_{T}^{B}} \sigma_{1}^{B} \nabla U \cdot \nabla \varphi_{B}+\int_{\Omega_{T}^{B}} I_{\text {ion }}(V, \widetilde{w}) \varphi_{B} \mathrm{~d} x \mathrm{~d} t \\
& \quad+\int_{\Omega_{T}^{B}}\left(\sigma_{1}^{B}+\sigma_{2}^{B}\right) \nabla U \cdot \nabla \varphi_{D}^{1} \mathrm{~d} x \mathrm{~d} t+\int_{\Omega_{T}^{B}} \sigma_{1}^{B} \nabla V \cdot \nabla \varphi_{D}^{1} \mathrm{~d} x \mathrm{~d} t \\
& \quad+\int_{\Omega_{T}^{D}} \sigma^{D} \nabla U \cdot \nabla \varphi_{D}^{2} \mathrm{~d} x \mathrm{~d} t-\alpha \int_{\Gamma_{T}}[U] \partial_{t}\left[\varphi_{D}\right] \mathrm{d} \sigma \mathrm{d} t+\beta \int_{\Gamma_{T}}[U]\left[\varphi_{D}\right] \mathrm{d} \sigma \mathrm{d} t \\
& =\int_{\Omega_{T}^{B}} f_{1} \varphi_{B} \mathrm{~d} x \mathrm{~d} t+\int_{\Omega_{T}^{B}}\left(f_{1}-f_{2}\right) \varphi_{D}^{1} \mathrm{~d} x \mathrm{~d} t,+\int_{\Omega^{B}} \bar{v}_{0} \varphi_{B}(0) \mathrm{d} x \\
& \quad+\alpha \int_{\Gamma} s_{0}\left[\varphi_{D}\right](0) \mathrm{d} \sigma,
\end{aligned}
$$

for every $\varphi_{B} \in L^{2}\left(0, T ; H_{\text {null }}^{1}\left(\Omega^{B}\right)\right) \cap H^{1}\left(0, T ; L^{2}\left(\Omega^{B}\right)\right), \varphi_{D} \in L^{2}\left(0, T ; \mathcal{X}_{0}^{1}(\Omega)\right)$ (which means $\varphi_{D}^{1} \in L^{2}\left(0, T ; H_{\text {null }}^{1}\left(\Omega^{B}\right)\right), \varphi_{D}^{2} \in L^{2}\left(0, T ; H_{\text {null }}^{1}\left(\Omega^{D}\right)\right)$ ), and $\left[\varphi_{D}\right] \in H^{1}\left(0, T ; L^{2}(\Gamma)\right)$, with $\varphi_{B}(T)=0$ and $\left[\varphi_{D}\right](T)=0$. Here, $\left[\varphi_{D}\right]=$ $\varphi_{D}^{1}-\varphi_{D}^{2}$ on $\Gamma$. We remark that the notation $\varphi_{D}^{1}$ for a test function acting in $\Omega^{B}$ is slightly counterintuitive, but it allows us to write concisely the jump of such a function across the interface. Clearly, by (2.25), we get the following energy inequality:

$$
\begin{aligned}
& \sup _{t \in(0, T)} \int_{\Omega^{B}} V^{2}(x, t) \mathrm{d} x+\int_{\Omega_{T}^{B}}|\nabla V+\nabla U|^{2} \mathrm{~d} x \mathrm{~d} t+\int_{\Omega_{T}^{B}}|\nabla U|^{2} \mathrm{~d} x \mathrm{~d} t \\
& \quad+\int_{\Omega_{T}^{D}}|\nabla U|^{2} \mathrm{~d} x \mathrm{~d} t+\sup _{t \in(0, T)} \int_{\Gamma}[U]^{2}(x, t) \mathrm{d} \sigma+\int_{\Gamma_{T}}[U]^{2} \mathrm{~d} \sigma \mathrm{d} t \\
& \leq \gamma\left(\left\|f_{1}\right\|_{L^{2}\left(\Omega_{T}^{B}\right)}^{2}+\left\|f_{2}\right\|_{L^{2}\left(\Omega_{T}^{B}\right)}^{2}+\left\|\bar{v}_{0}\right\|_{L^{2}\left(\Omega^{B}\right)}^{2}+\left\|s_{0}\right\|_{L^{2}(\Gamma)}^{2}+1\right),
\end{aligned}
$$

where $\gamma$ depends on $\gamma_{0}, \gamma_{I}, \alpha, \beta$, and the geometry. Notice that, by (2.36), it follows also that:

$$
\begin{aligned}
& \int_{\Omega_{T}^{B}}|\nabla V|^{2} \mathrm{~d} x \mathrm{~d} t \leq \gamma\left(\left\|f_{1}\right\|_{L^{2}\left(\Omega_{T}^{B}\right)}^{2}+\left\|f_{2}\right\|_{L^{2}\left(\Omega_{T}^{B}\right)}^{2}\right. \\
& \left.\quad+\left\|\bar{v}_{0}\right\|_{L^{2}\left(\Omega^{B}\right)}^{2}+\left\|s_{0}\right\|_{L^{2}(\Gamma)}^{2}+1\right) .
\end{aligned}
$$




\section{Well-Posedness}

We will consider our problem in an abstract setting, and to this purpose, we need first "to move" the source $f_{1}-f_{2}$ from $(2.27)$ to $(2.26),(2.31)$, and (2.34). Then, we introduce a bilinear form $a$ on $W \times W$, such that the problem is reduced to the abstract scheme (3.47). This form is constructed with auxiliary functions which are obtained in Proposition 3.1 and in Remark 3.2. In Proposition 3.3, we prove the necessary properties of the form $a$. In Theorem 3.4 and Proposition 3.5, we prove existence of solutions to the problem with $I_{\text {ion }} \equiv 0$. Finally, the full result is obtained in Theorem 3.6, where the complete problem will be treated as a nonlinear perturbation of this case (see, for instance, $[9,13,16,22]$ ).

We start by considering, for a.e. $t \in(0, T)$, the following auxiliary problem:

$$
\begin{gathered}
-\operatorname{div}\left(\left(\sigma_{1}^{B}+\sigma_{2}^{B}\right) \nabla \widetilde{u}\right)=f_{1}-f_{2}, \quad \text { in } \quad \Omega^{B} \\
\sigma_{1}^{B} \nabla \widetilde{u} \cdot \nu=0, \quad \text { on } \quad \Gamma ; \\
\widetilde{u}=0, \quad \text { on } \quad \partial \Omega^{B} \cap \partial \Omega .
\end{gathered}
$$

Clearly, problem (3.1)-(3.3) is classical and admits a unique solution $\widetilde{u} \in$ $H^{1}\left(0, T ; H_{\text {null }}^{1}\left(\Omega^{B}\right)\right)$. Moreover, we extend $\widetilde{u}$ inside $\Omega^{D}$ by zero, so that it has a nonzero jump on $\Gamma$; i.e., $\widetilde{u} \in \mathcal{X}_{0}^{1}(\Omega)$. On $\Gamma$, let us define

$$
q:=-\alpha \frac{\partial[\widetilde{u}]}{\partial t}-\beta[\widetilde{u}]
$$

and consider the problem for $(v, u) \in L^{2}\left(0, T ; H_{\text {null }}^{1}\left(\Omega^{B}\right)\right) \times L^{2}\left(0, T ; \mathcal{X}_{0}^{1}(\Omega)\right)$ given by

$$
\begin{gathered}
\frac{\partial v}{\partial t}-\operatorname{div}\left(\sigma_{1}^{B} \nabla v\right)=f_{1}+\operatorname{div}\left(\sigma_{1}^{B} \nabla u\right)+\operatorname{div}\left(\sigma_{1}^{B} \nabla \widetilde{u}\right), \text { in } \Omega_{T}^{B} ; \\
-\operatorname{div}\left(\left(\sigma_{1}^{B}+\sigma_{2}^{B}\right) \nabla u\right)=\operatorname{div}\left(\sigma_{1}^{B} \nabla v\right), \quad \text { in } \quad \Omega_{T}^{B} ; \\
-\operatorname{div}\left(\sigma^{D} \nabla u\right)=0, \quad \text { in } \quad \Omega_{T}^{D} ; \\
\sigma_{1}^{B} \nabla(v+u) \cdot \nu=0, \quad \text { on } \quad \Gamma_{T} ; \\
\sigma_{2}^{B} \nabla u \cdot \nu=\sigma^{D} \nabla u \cdot \nu, \quad \text { on } \quad \Gamma_{T} ; \\
\alpha \frac{\partial}{\partial t}[u]+\beta[u]=\sigma_{2}^{B} \nabla u \cdot \nu+q, \text { on } \Gamma_{T} ; \\
v, u=0, \quad \text { on } \partial \Omega \times(0, T) ; \\
v(x, 0)=\bar{v}_{0}(x), \text { on } \Omega^{B} ; \\
{[u](x, 0)=s_{0}(x)-[\widetilde{u}](x, 0), \quad \text { on } \quad \Gamma .}
\end{gathered}
$$


The weak formulation of the previous problem is given by

$$
\begin{aligned}
& -\int_{\Omega_{T}^{B}} v \partial_{t} \varphi_{B} \mathrm{~d} x \mathrm{~d} t+\int_{\Omega_{T}^{B}} \sigma_{1}^{B} \nabla v \cdot \nabla \varphi_{B} \mathrm{~d} x \mathrm{~d} t+\int_{\Omega_{T}^{B}} \sigma_{1}^{B} \nabla u \cdot \nabla \varphi_{B} \mathrm{~d} x \mathrm{~d} t \\
& \quad+\int_{\Omega_{T}^{B}}\left(\sigma_{1}^{B}+\sigma_{2}^{B}\right) \nabla u \cdot \nabla \varphi_{D}^{1} \mathrm{~d} x \mathrm{~d} t+\int_{\Omega_{T}^{B}} \sigma_{1}^{B} \nabla v \cdot \nabla \varphi_{D}^{1} \mathrm{~d} x \mathrm{~d} t \\
& \quad+\int_{\Omega_{T}^{D}} \sigma^{D} \nabla u \cdot \nabla \varphi_{D}^{2} \mathrm{~d} x \mathrm{~d} t-\alpha \int_{\Gamma_{T}}[u] \partial_{t}\left[\varphi_{D}\right] \mathrm{d} \sigma \mathrm{d} t+\beta \int_{\Gamma_{T}}[u]\left[\varphi_{D}\right] \mathrm{d} \sigma \mathrm{d} t \\
& =\int_{\Omega_{T}^{B}} f_{1} \varphi_{B} \mathrm{~d} x \mathrm{~d} t-\int_{\Omega_{T}^{B}} \sigma_{1}^{B} \nabla \widetilde{u} \cdot \nabla \varphi_{B} \mathrm{~d} x \mathrm{~d} t+\int_{\Gamma_{T}} q\left[\varphi_{D}\right] \mathrm{d} \sigma \mathrm{d} t,
\end{aligned}
$$

for every $\varphi_{B} \in L^{2}\left(0, T ; H_{\text {null }}^{1}\left(\Omega^{B}\right)\right) \cap H_{0}^{1}\left(0, T ; L^{2}\left(\Omega^{B}\right)\right), \varphi_{D} \in L^{2}\left(0, T ; \mathcal{X}_{0}^{1}(\Omega)\right)$, where, as before, $\left[\varphi_{D}\right]=\varphi_{D}^{1}-\varphi_{D}^{2}$ and $\left[\varphi_{D}\right] \in H_{0}^{1}\left(0, T ; L^{2}(\Gamma)\right)$. The weak formulation (3.14) shall be complemented with the initial conditions. Indeed, as it will be proved in Theorem 3.4, we have $v \in \mathcal{C}^{0}\left([0, T] ; L^{2}\left(\Omega^{B}\right)\right)$ and $[u] \in \mathcal{C}^{0}\left([0, T] ; L^{2}(\Gamma)\right)$.

The next step is to define a suitable bilinear form on $W$, which is continuous and coercive. To this purpose, we need the following result.

Proposition 3.1. Let $(w, r) \in W$ be assigned. Then, there exists a unique solution $\mathcal{W} \in \mathcal{X}_{0}^{1}(\Omega)$ of the problem

$$
\begin{gathered}
-\operatorname{div}\left(\left(\sigma_{1}^{B}+\sigma_{2}^{B}\right) \nabla \mathcal{W}\right)=\operatorname{div}\left(\sigma_{1}^{B} \nabla w\right), \quad \text { in } \quad \Omega^{B} ; \\
-\operatorname{div}\left(\sigma^{D} \nabla \mathcal{W}\right)=0, \quad \text { in } \quad \Omega^{D} ; \\
\left(\sigma_{1}^{B}+\sigma_{2}^{B}\right) \nabla \mathcal{W} \cdot \nu=\sigma^{D} \nabla \mathcal{W} \cdot \nu-\sigma_{1}^{B} \nabla w \cdot \nu, \quad \text { on } \quad \Gamma ; \\
{[\mathcal{W}]=r, \quad \text { on } \quad \Gamma ;} \\
\mathcal{W}=0, \quad \text { on } \quad \partial \Omega .
\end{gathered}
$$

Moreover, there exists a constant $\gamma>0$, depending on $\sigma_{1}^{B}, \sigma_{2}^{B}, \sigma^{D}$, and the geometry, such that

$$
\|\mathcal{W}\|_{\mathcal{X}_{0}^{1}(\Omega)} \leq \gamma\left(\|w\|_{H^{1}\left(\Omega^{B}\right)}+\|r\|_{H^{1 / 2}(\Gamma)}\right) .
$$

Proof. Uniqueness for problem (3.15)-(3.19) is a straightforward consequence of its linearity. To prove that a solution does exist, we first consider the following auxiliary problem:

$$
\begin{aligned}
-\operatorname{div}\left(\left(\sigma_{1}^{B}+\sigma_{2}^{B}\right) \nabla \overline{\mathcal{W}}_{1}\right) & =\operatorname{div}\left(\sigma_{1}^{B} \nabla w\right), \text { in } \Omega^{B} ; \\
\overline{\mathcal{W}}_{1} & =r, \text { on } \quad \Gamma ; \\
\overline{\mathcal{W}}_{1} & =0, \text { in } \Omega^{D} ; \\
\overline{\mathcal{W}}_{1} & =0, \text { on } \partial \Omega .
\end{aligned}
$$

Clearly, the previous problem admits a unique solution $\overline{\mathcal{W}}_{1} \in \mathcal{X}_{0}^{1}(\Omega)$. Moreover, there exists a constant $\gamma>0$, depending on $\sigma_{1}^{B}, \sigma_{2}^{B}$ and the geometry, such that

$$
\left\|\overline{\mathcal{W}}_{1}\right\|_{\mathcal{X}_{0}^{1}(\Omega)} \leq \gamma\left(\|w\|_{H^{1}\left(\Omega^{B}\right)}+\|r\|_{H^{1 / 2}(\Gamma)}\right) .
$$

Indeed, let us denote by $\widetilde{r} \in H_{\text {null }}^{1}\left(\Omega^{B}\right)$ an extension of $r$ from $\Gamma$ to the whole 
$\Omega^{B}$, such that $\|\widetilde{r}\|_{H^{1}\left(\Omega^{B}\right)} \leq \gamma\|r\|_{H^{1 / 2}(\Gamma)}$, and set $\overline{\mathcal{W}}_{1}^{r}:=\overline{\mathcal{W}}_{1}-\widetilde{r}$. Clearly, $\overline{\mathcal{W}}_{1}^{r}$ satisfies the problem

$-\operatorname{div}\left(\left(\sigma_{1}^{B}+\sigma_{2}^{B}\right) \nabla \overline{\mathcal{W}}_{1}^{r}\right)=\operatorname{div}\left(\sigma_{1}^{B} \nabla w\right)+\operatorname{div}\left(\left(\sigma_{1}^{B}+\sigma_{2}^{B}\right) \nabla \widetilde{r}\right), \quad$ in $\quad \Omega^{B} ;$

$$
\overline{\mathcal{W}}_{1}^{r}=0, \quad \text { on } \quad \partial \Omega^{B} .
$$

Therefore, by the standard energy inequality, we get

$$
\int_{\Omega^{B}}\left|\nabla \overline{\mathcal{W}}_{1}^{r}\right|^{2} \mathrm{~d} x \leq \gamma\left(\|\nabla w\|_{L^{2}\left(\Omega^{B}\right)}^{2}+\|r\|_{H^{1 / 2}(\Gamma)}^{2}\right),
$$

which implies (3.25).

Now, let us consider the second auxiliary problem for $\overline{\mathcal{W}}_{2} \in H_{0}^{1}(\Omega)$ given by

$$
\begin{gathered}
-\operatorname{div}\left(\left(\sigma_{1}^{B}+\sigma_{2}^{B}\right) \nabla \overline{\mathcal{W}}_{2}\right)=0, \text { in } \Omega^{B} ; \\
-\operatorname{div}\left(\sigma^{D} \nabla \overline{\mathcal{W}}_{2}\right)=0, \text { in } \Omega^{D} ; \\
{\left[\overline{\mathcal{W}}_{2}\right]=0, \text { on } \Gamma ;} \\
\left(\left(\sigma_{1}^{B}+\sigma_{2}^{B}\right) \nabla \overline{\mathcal{W}}_{2}-\sigma^{D} \nabla \overline{\mathcal{W}}_{2}\right) \cdot \nu=-\left(\left(\sigma_{1}^{B}+\sigma_{2}^{B}\right) \nabla \overline{\mathcal{W}}_{1}+\sigma_{1}^{B} \nabla w\right) \cdot \nu, \\
\overline{\mathcal{W}}_{2}=0, \text { on } \quad \partial \Omega .
\end{gathered}
$$

Existence and uniqueness for the previous problem are guaranteed by [1, Lemma 5]; moreover, the weak formulation of (3.29)-(3.33) is given by

$$
\begin{aligned}
0= & \int_{\Omega^{B}}\left(\sigma_{1}^{B}+\sigma_{2}^{B}\right) \nabla \overline{\mathcal{W}}_{2} \cdot \nabla \varphi \mathrm{d} x+\int_{\Gamma}\left(\sigma_{1}^{B}+\sigma_{2}^{B}\right) \nabla \overline{\mathcal{W}}_{2} \cdot \nu \varphi \mathrm{d} \sigma \\
& +\int_{\Omega^{D}} \sigma^{D} \nabla \overline{\mathcal{W}}_{2} \cdot \nabla \varphi \mathrm{d} x-\int_{\Gamma} \sigma^{D} \nabla \overline{\mathcal{W}}_{2} \cdot \nu \varphi \mathrm{d} \sigma \\
= & \int_{\Omega^{B}}\left(\sigma_{1}^{B}+\sigma_{2}^{B}\right) \nabla \overline{\mathcal{W}}_{2} \cdot \nabla \varphi \mathrm{d} x+\int_{\Omega^{D}} \sigma^{D} \nabla \overline{\mathcal{W}}_{2} \cdot \nabla \varphi \mathrm{d} x \\
& -\int_{\Gamma}\left(\sigma_{1}^{B}+\sigma_{2}^{B}\right) \nabla \overline{\mathcal{W}}_{1} \cdot \nu \varphi \mathrm{d} \sigma-\int_{\Gamma} \sigma_{1}^{B} \nabla w \cdot \nu \varphi \mathrm{d} \sigma,
\end{aligned}
$$

for every $\varphi \in H_{0}^{1}(\Omega)$. From (3.21), we obtain

$$
\begin{aligned}
& -\int_{\Gamma}\left(\sigma_{1}^{B}+\sigma_{2}^{B}\right) \nabla \overline{\mathcal{W}}_{1} \cdot \nu \varphi \mathrm{d} \sigma-\int_{\Gamma} \sigma_{1}^{B} \nabla w \cdot \nu \varphi \mathrm{d} \sigma \\
& =\int_{\Omega^{B}}\left(\sigma_{1}^{B}+\sigma_{2}^{B}\right) \nabla \overline{\mathcal{W}}_{1} \cdot \nabla \varphi \mathrm{d} x+\int_{\Omega^{B}} \sigma_{1}^{B} \nabla w \cdot \nabla \varphi \mathrm{d} x,
\end{aligned}
$$

which, replaced in (3.34), provides

$$
\begin{aligned}
0=\int_{\Omega^{B}} & \left(\sigma_{1}^{B}+\sigma_{2}^{B}\right) \nabla \overline{\mathcal{W}}_{2} \cdot \nabla \varphi \mathrm{d} x+\int_{\Omega^{D}} \sigma^{D} \nabla \overline{\mathcal{W}}_{2} \cdot \nabla \varphi \mathrm{d} x \\
& +\int_{\Omega^{B}}\left(\sigma_{1}^{B}+\sigma_{2}^{B}\right) \nabla \overline{\mathcal{W}}_{1} \cdot \nabla \varphi \mathrm{d} x+\int_{\Omega^{B}} \sigma_{1}^{B} \nabla w \cdot \nabla \varphi \mathrm{d} x .
\end{aligned}
$$


By taking $\varphi=\overline{\mathcal{W}}_{2}$ in $(3.35)$, we get

$$
\begin{aligned}
& \int_{\Omega^{B}}\left(\sigma_{1}^{B}+\sigma_{2}^{B}\right)\left|\nabla \overline{\mathcal{W}}_{2}\right|^{2} \mathrm{~d} x+\int_{\Omega^{D}} \sigma^{D}\left|\nabla \overline{\mathcal{W}}_{2}\right|^{2} \mathrm{~d} x \\
& =-\int_{\Omega^{B}}\left(\sigma_{1}^{B}+\sigma_{2}^{B}\right) \nabla \overline{\mathcal{W}}_{1} \cdot \nabla \overline{\mathcal{W}}_{2} \mathrm{~d} x-\int_{\Omega^{B}} \sigma_{1}^{B} \nabla w \cdot \nabla \overline{\mathcal{W}}_{2} \mathrm{~d} x,
\end{aligned}
$$

which, taking into account (3.25), implies

$$
\begin{aligned}
\left\|\nabla \overline{\mathcal{W}}_{2}\right\|_{L^{2}(\Omega)} & \leq \gamma\left(\left\|\nabla \overline{\mathcal{W}}_{1}\right\|_{L^{2}\left(\Omega^{B}\right)}+\|\nabla w\|_{L^{2}\left(\Omega^{B}\right)}\right) \\
& \leq \gamma\left(\|w\|_{H^{1}\left(\Omega^{B}\right)}+\|r\|_{H^{1 / 2}(\Gamma)}\right)
\end{aligned}
$$

with $\gamma$ depending only on $\sigma_{1}^{B}, \sigma_{2}^{B}, \sigma^{D}$ and the geometry.

Finally, setting $\mathcal{W}=\overline{\mathcal{W}}_{1}+\overline{\mathcal{W}}_{2}$, it is easy to see that $\mathcal{W} \in \mathcal{X}_{0}^{1}(\Omega)$ and satisfies (3.15)-(3.19) and also (3.20).

Remark 3.2. We point out that, taking $\widetilde{r} \in H_{\text {null }}^{1}\left(\Omega^{B}\right)$ as in the proof of Proposition 3.1 and defining

$$
\mathcal{W}^{r}= \begin{cases}\mathcal{W}-\tilde{r}, & \text { in } \Omega^{B} \\ \mathcal{W}, \quad \text { in } & \Omega^{D}\end{cases}
$$

we get that $\mathcal{W}^{r} \in H_{0}^{1}(\Omega)$ and satisfies

$$
-\operatorname{div}\left(\left(\sigma_{1}^{B}+\sigma_{2}^{B}\right) \nabla \mathcal{W}^{r}\right)=\operatorname{div}\left(\sigma_{1}^{B} \nabla w\right)+\operatorname{div}\left(\left(\sigma_{1}^{B}+\sigma_{2}^{B}\right) \nabla \widetilde{r}\right), \quad \text { in } \quad \Omega^{B}
$$

$$
-\operatorname{div}\left(\sigma^{D} \nabla \mathcal{W}^{r}\right)=0, \quad \text { in } \quad \Omega^{D}
$$

$\left(\sigma_{1}^{B}+\sigma_{2}^{B}\right) \nabla \mathcal{W}^{r} \cdot \nu=\sigma^{D} \nabla \mathcal{W}^{r} \cdot \nu-\sigma_{1}^{B} \nabla w \cdot \nu-\left(\sigma_{1}^{B}+\sigma_{2}^{B}\right) \nabla \widetilde{r} \cdot \nu, \quad$ on $\quad \Gamma$

$$
\begin{gathered}
{\left[\mathcal{W}^{r}\right]=0, \quad \text { on } \quad \Gamma ;} \\
\mathcal{W}^{r}=0, \quad \text { on } \quad \partial \Omega .
\end{gathered}
$$

The weak formulation of problem (3.38)-(3.41) is given by

$$
\begin{aligned}
& \int_{\Omega^{B}}\left(\sigma_{1}^{B}+\sigma_{2}^{B}\right) \nabla \mathcal{W}^{r} \cdot \nabla \phi \mathrm{d} x+\int_{\Omega^{D}} \sigma^{D} \nabla \mathcal{W}^{r} \cdot \nabla \phi \mathrm{d} x \\
& =-\int_{\Omega^{B}} \sigma_{1}^{B} \nabla w \cdot \nabla \phi \mathrm{d} x-\int_{\Omega^{B}}\left(\sigma_{1}^{B}+\sigma_{2}^{B}\right) \nabla \widetilde{r} \cdot \nabla \phi \mathrm{d} x,
\end{aligned}
$$


for every $\phi \in H_{0}^{1}(\Omega)$, which implies that

$$
\begin{aligned}
& \int_{\Omega^{B}}\left(\sigma_{1}^{B}+\sigma_{2}^{B}\right) \nabla \mathcal{W} \cdot \nabla \phi \mathrm{d} x+\int_{\Omega^{D}} \sigma^{D} \nabla \mathcal{W} \cdot \nabla \phi \mathrm{d} x \\
& =-\int_{\Omega^{B}} \sigma_{1}^{B} \nabla w \cdot \nabla \phi \mathrm{d} x,
\end{aligned}
$$

for every $\phi \in H_{0}^{1}(\Omega)$.

Now, we are in the position to define the bilinear form $a: W \times W \rightarrow \mathbb{R}$ as

$$
\begin{aligned}
& a((w, r),(\bar{w}, s)):=\int_{\Omega^{B}} \sigma_{1}^{B} \nabla w \cdot \nabla \bar{w} \mathrm{~d} x+\int_{\Omega^{B}} \sigma_{1}^{B} \nabla \mathcal{W} \cdot \nabla \bar{w} \mathrm{~d} x \\
& \quad+\int_{\Omega^{B}}\left(\sigma_{1}^{B}+\sigma_{2}^{B}\right) \nabla \mathcal{W} \cdot \nabla \overline{\mathcal{W}} \mathrm{d} x+\int_{\Omega^{B}} \sigma_{1}^{B} \nabla w \cdot \nabla \overline{\mathcal{W}} \mathrm{d} x \\
& \quad+\int_{\Omega^{D}} \sigma^{D} \nabla \mathcal{W} \cdot \nabla \overline{\mathcal{W}} \mathrm{d} x+\beta \int_{\Gamma} r s \mathrm{~d} \sigma,
\end{aligned}
$$

where $\mathcal{W}$ and $\overline{\mathcal{W}}$ are the solutions of (3.15)-(3.19) corresponding to $(w, r)$ and $(\bar{w}, s)$, respectively.

Proposition 3.3. The bilinear form $a: W \times W \rightarrow \mathbb{R}$, defined in (3.45), is symmetric, continuous, and coercive.

Proof. Notice that the bilinear form $a$ can be rewritten as

$$
\begin{aligned}
a( & (w, r),(\bar{w}, s)) \\
= & \int_{\Omega^{B}} \sigma_{1}^{B} \nabla(w+\mathcal{W}) \cdot \nabla \bar{w} \mathrm{~d} x+\int_{\Omega^{B}} \sigma_{1}^{B} \nabla(w+\mathcal{W}) \cdot \nabla \overline{\mathcal{W}} \mathrm{d} x \\
& +\int_{\Omega^{B}} \sigma_{2}^{B} \nabla \mathcal{W} \cdot \nabla \overline{\mathcal{W}} \mathrm{d} x+\int_{\Omega^{D}} \sigma^{D} \nabla \mathcal{W} \cdot \nabla \overline{\mathcal{W}} \mathrm{d} x+\beta \int_{\Gamma} r s \mathrm{~d} \sigma \\
= & \int_{\Omega^{B}} \sigma_{1}^{B} \nabla(w+\mathcal{W}) \cdot \nabla(\bar{w}+\overline{\mathcal{W}}) \mathrm{d} x \\
& +\int_{\Omega^{B}} \sigma_{2}^{B} \nabla \mathcal{W} \cdot \nabla \overline{\mathcal{W}} \mathrm{d} x+\int_{\Omega^{D}} \sigma^{D} \nabla \mathcal{W} \cdot \nabla \overline{\mathcal{W}} \mathrm{d} x+\beta \int_{\Gamma} r s \mathrm{~d} \sigma,
\end{aligned}
$$

which immediately proves that it is symmetric. Moreover, from (3.20), it easily follows that $a$ is continuous. To prove that it is also coercive, we note that

$$
\begin{aligned}
a((w, r),(w, r))= & \int_{\Omega^{B}} \sigma_{1}^{B} \nabla(w+\mathcal{W}) \cdot \nabla(w+\mathcal{W}) \mathrm{d} x+\int_{\Omega^{B}} \sigma_{2}^{B} \nabla \mathcal{W} \cdot \nabla \mathcal{W} \mathrm{d} x \\
& +\int_{\Omega^{D}} \sigma^{D} \nabla \mathcal{W} \cdot \nabla \mathcal{W} \mathrm{d} x+\beta \int_{\Gamma} r^{2} \mathrm{~d} \sigma \\
\geq & \gamma\left(\|\nabla w+\nabla \mathcal{W}\|_{L^{2}\left(\Omega^{B}\right)}^{2}+\|\nabla \mathcal{W}\|_{L^{2}\left(\Omega^{B}\right)}^{2}\right. \\
& \left.+\|\nabla \mathcal{W}\|_{L^{2}\left(\Omega^{D}\right)}^{2}+\|r\|_{L^{2}(\Gamma)}^{2}\right) \\
\geq & \gamma\left(\|w\|_{H^{1}\left(\Omega^{B}\right)}^{2}+\|r\|_{H^{1 / 2}(\Gamma)}^{2}\right),
\end{aligned}
$$


where, in the last inequality, we take into account that $r=[\mathcal{W}]($ see $(3.18))$ and we use the Poincaré inequality (2.7) and the classical trace inequality, which assure that

$\|r\|_{H^{1 / 2}(\Gamma)}^{2} \leq \gamma\|\mathcal{W}\|_{\mathcal{X}_{0}^{1}\left(\Omega^{B}\right)}^{2} \leq \gamma\left(\|\nabla \mathcal{W}\|_{L^{2}\left(\Omega^{B}\right)}^{2}+\|\nabla \mathcal{W}\|_{L^{2}\left(\Omega^{D}\right)}^{2}+\|r\|_{L^{2}(\Gamma)}^{2}\right)$.

Theorem 3.4. Assume that $\sigma_{1}^{B}, \sigma_{2}^{B}, \sigma^{D}, \alpha, \beta, f_{1}, f_{2}, \bar{v}_{0}, s_{0}$ are as in Sect. 2.3. Let $\widetilde{u}$ be the unique solution of problem (3.1)-(3.3), extended by zero inside $\Omega^{D}$, and $q$ be defined in (3.4). Then, problem (3.5)-(3.13) admits a unique solution $(v, u) \in L^{2}\left(0, T ; H_{\text {null }}^{1}\left(\Omega^{B}\right)\right) \times L^{2}\left(0, T ; \mathcal{X}_{0}^{1}(\Omega)\right)$, such that $v \in \mathcal{C}^{0}\left([0, T] ; L^{2}\left(\Omega^{B}\right)\right)$ and $[u] \in \mathcal{C}^{0}\left([0, T] ; L^{2}(\Gamma)\right)$.

Proof. Let us denote by $\langle\cdot, \cdot\rangle_{W^{*} W}$ the duality pairing between $W$ and its dual space $W^{*}$ and define $B \in W^{*}$ as

$$
\left\langle B,\left(\varphi_{B},\left[\varphi_{D}\right]\right)\right\rangle_{W^{*} W}=\int_{\Omega^{B}} f_{1} \varphi_{B} \mathrm{~d} x-\int_{\Omega^{B}} \sigma_{1}^{B} \nabla \widetilde{u} \cdot \nabla \varphi_{B} \mathrm{~d} x+\int_{\Gamma} q\left[\varphi_{D}\right] \mathrm{d} \sigma,
$$

for every $\left(\varphi_{B},\left[\varphi_{D}\right]\right) \in W$. Using the bilinear form $a$ introduced in (3.45), we can consider the following abstract problem:

$$
\begin{aligned}
& \text { find }(v,[u]) \in L^{2}(0, T ; W) \cap \mathcal{C}^{0}([0, T] ; H) \\
& \qquad \text { with } v(0)=\bar{v}_{0} \text { and }[u](0)=s_{0}-[\widetilde{u}](0) \text { such that } \\
& \begin{array}{l}
\frac{\mathrm{d}}{\mathrm{d} t}\left((v,[u]),\left(\varphi_{B},\left[\varphi_{D}\right]\right)\right)_{H}+a\left((v,[u]),\left(\varphi_{B},\left[\varphi_{D}\right]\right)\right) \\
\quad=\left\langle B,\left(\varphi_{B},\left[\varphi_{D}\right]\right)\right\rangle_{W^{*} W}, \quad \forall\left(\varphi_{B},\left[\varphi_{D}\right]\right) \in W,
\end{array}
\end{aligned}
$$

in the distributional sense. By [25, Theorem 23.A], the problem (3.47) is well-posed and it is not difficult to see that its weak formulation is given by

$$
\begin{aligned}
& -\int_{\Omega_{T}^{B}} v \varphi_{B} \partial_{t} \psi \mathrm{d} x \mathrm{~d} t+\int_{\Omega_{T}^{B}} \sigma_{1}^{B} \nabla v \cdot \nabla \varphi_{B} \psi \mathrm{d} x \mathrm{~d} t+\int_{\Omega_{T}^{B}} \sigma_{1}^{B} \nabla u \cdot \nabla \varphi_{B} \psi \mathrm{d} x \mathrm{~d} t \\
& \quad+\int_{\Omega_{T}^{B}}\left(\sigma_{1}^{B}+\sigma_{2}^{B}\right) \nabla u \cdot \nabla \varphi_{D}^{1} \psi \mathrm{d} x \mathrm{~d} t+\int_{\Omega_{T}^{B}} \sigma_{1}^{B} \nabla v \cdot \nabla \varphi_{D}^{1} \psi \mathrm{d} x \mathrm{~d} t \\
& \quad+\int_{\Omega_{T}^{D}} \sigma^{D} \nabla u \cdot \nabla \varphi_{D}^{2} \psi \mathrm{d} x \mathrm{~d} t-\alpha \int_{\Gamma_{T}}[u]\left[\varphi_{D}\right] \partial_{t} \psi \mathrm{d} \sigma \mathrm{d} t+\beta \int_{\Gamma_{T}}[u]\left[\varphi_{D}\right] \psi \mathrm{d} \sigma \mathrm{d} t \\
& =\int_{\Omega_{T}^{B}} f_{1} \varphi_{B} \psi \mathrm{d} x \mathrm{~d} t-\int_{\Omega_{T}^{B}} \sigma_{1}^{B} \nabla \widetilde{u} \cdot \nabla \varphi_{B} \psi \mathrm{d} x \mathrm{~d} t+\int_{\Gamma_{T}} q\left[\varphi_{D}\right] \psi \mathrm{d} \sigma \mathrm{d} t,
\end{aligned}
$$

for every $\left(\varphi_{B},\left[\varphi_{D}\right]\right) \in W$ and every $\psi \in \mathcal{C}_{0}^{\infty}(0, T)$, where $\varphi_{D}$ inside $\Omega^{B}$ and $\Omega^{D}$ is defined as the solution of (3.15)-(3.19), starting from $\varphi_{B} \in H_{\text {null }}^{1}\left(\Omega^{B}\right)$ and $\left[\varphi_{D}\right] \in H_{0}^{1 / 2}(\Gamma, \Omega)$. Clearly, (3.48) shall be complemented with the initial conditions.

Notice that (3.48) formally coincides with (3.14); however, in (3.14), the test function $\varphi_{D}$ is a generic function belonging to $L^{2}\left(0, T ; \mathcal{X}_{0}^{1}(\Omega)\right)$, while in the present case, it is the solution of an assigned differential problem. Hence, to state that, actually, the two weak formulations are equivalent, we have to prove that we can replace the prescribed $\varphi_{D}$ in (3.48) with a generic test 
function belonging to $\mathcal{X}_{0}^{1}(\Omega)$. To this purpose, let us fix $\left[\varphi_{D}\right] \in H_{0}^{1 / 2}(\Gamma, \Omega)$ and choose two generic functions $\varphi^{1} \in H_{\text {null }}^{1}\left(\Omega^{B}\right)$ and $\varphi^{2} \in H_{\text {null }}^{1}\left(\Omega^{D}\right)$, such that $[\varphi]:=\varphi^{1}-\varphi^{2}=\left[\varphi_{D}\right]$. By (3.44), with $\phi=\varphi_{D}-\varphi \in H_{0}^{1}(\Omega)$, we get

$$
\begin{aligned}
& \int_{\Omega_{T}^{B}}\left(\sigma_{1}^{B}+\sigma_{2}^{B}\right) \nabla u \cdot \nabla \varphi_{D}^{1} \psi \mathrm{d} x \mathrm{~d} t+\int_{\Omega_{T}^{B}} \sigma_{1}^{B} \nabla v \cdot \nabla \varphi_{D}^{1} \psi \mathrm{d} x \mathrm{~d} t \\
& \quad+\int_{\Omega_{T}^{D}} \sigma^{D} \nabla u \cdot \nabla \varphi_{D}^{2} \psi \mathrm{d} x \mathrm{~d} t \\
& =\int_{\Omega_{T}^{B}}\left(\sigma_{1}^{B}+\sigma_{2}^{B}\right) \nabla u \cdot \nabla \varphi^{1} \psi \mathrm{d} x \mathrm{~d} t+\int_{\Omega_{T}^{B}} \sigma_{1}^{B} \nabla v \cdot \nabla \varphi^{1} \psi \mathrm{d} x \mathrm{~d} t \\
& \quad+\int_{\Omega_{T}^{D}} \sigma^{D} \nabla u \cdot \nabla \varphi^{2} \psi \mathrm{d} x \mathrm{~d} t .
\end{aligned}
$$

Hence, replacing (3.49) in (3.48), it follows that it is possible to take $\varphi_{D} \in$ $\mathcal{X}_{0}^{1}(\Omega)$ arbitrarily in (3.48) and, thus, such a weak formulation coincides with (3.14), once we take into account the density of product functions in $L^{2}\left(0, T ; H_{\text {null }}^{1}\left(\Omega^{B}\right)\right)$ and in $L^{2}\left(0, T ; \mathcal{X}_{0}^{1}(\Omega)\right)$.

Proposition 3.5. Assume that $\sigma_{1}^{B}, \sigma_{2}^{B}, \sigma^{D}, \alpha, \beta, f_{1}, f_{2}, \bar{v}_{0}, s_{0}$ are as in Sect. 2.3. Assume that $I_{\mathrm{ion}} \equiv 0$. Then, problem (2.26)-(2.34) admits a unique solution $(V, U) \in L^{2}\left(0, T ; H_{\text {null }}^{1}\left(\Omega^{B}\right)\right) \times L^{2}\left(0, T ; \mathcal{X}_{0}^{1}(\Omega)\right)$, such that $V \in \mathcal{C}^{0}([0, T]$; $\left.L^{2}\left(\Omega^{B}\right)\right)$ and $[U] \in \mathcal{C}^{0}\left([0, T] ; L^{2}(\Gamma)\right)$.

Proof. Uniqueness easily follows by the linearity of problem $(2.26)-(2.34)$. To prove existence, set $V=v$ and $U=u+\widetilde{u}$, where $\widetilde{u} \in H^{1}\left(0, T ; \mathcal{X}_{0}^{1}(\Omega)\right)$ is the solution of problem (3.1)-(3.3) and the pair $(v, u) \in L^{2}\left(0, T ; H_{\text {null }}^{1}\left(\Omega^{B}\right)\right) \times$ $L^{2}\left(0, T ; \mathcal{X}_{0}^{1}(\Omega)\right)$, with $v \in \mathcal{C}^{0}\left([0, T] ; L^{2}\left(\Omega^{B}\right)\right)$ and $[u] \in \mathcal{C}^{0}\left([0, T] ; L^{2}(\Gamma)\right)$, is the solution of (3.5)-(3.13), whose existence is guaranteed by Proposition 3.4. Then, by (3.14), we get

$$
\begin{aligned}
& -\int_{\Omega_{T}^{B}} V \partial_{t} \varphi_{B} \mathrm{~d} x \mathrm{~d} t+\int_{\Omega_{T}^{B}} \sigma_{1}^{B} \nabla V \cdot \nabla \varphi_{B} \mathrm{~d} x \mathrm{~d} t+\int_{\Omega_{T}^{B}} \sigma_{1}^{B} \nabla U \cdot \nabla \varphi_{B} \mathrm{~d} x \mathrm{~d} t \\
& \quad+\int_{\Omega_{T}^{B}}\left(\sigma_{1}^{B}+\sigma_{2}^{B}\right) \nabla U \cdot \nabla \varphi_{D}^{1} \mathrm{~d} x \mathrm{~d} t-\int_{\Omega_{T}^{B}}\left(\sigma_{1}^{B}+\sigma_{2}^{B}\right) \nabla \widetilde{u} \cdot \nabla \varphi_{D}^{1} \mathrm{~d} x \mathrm{~d} t \\
& \quad+\int_{\Omega_{T}^{B}} \sigma_{1}^{B} \nabla V \cdot \nabla \varphi_{D}^{1} \mathrm{~d} x \mathrm{~d} t \\
& \quad+\int_{\Omega_{T}^{D}} \sigma^{D} \nabla U \cdot \nabla \varphi_{D}^{2} \mathrm{~d} x \mathrm{~d} t-\alpha \int_{\Gamma_{T}}[U] \partial_{t}\left[\varphi_{D}\right] \mathrm{d} \sigma \mathrm{d} t+\beta \int_{\Gamma_{T}}[U]\left[\varphi_{D}\right] \mathrm{d} \sigma \mathrm{d} t \\
& =\int_{\Omega_{T}^{B}} f_{1} \varphi_{B} \mathrm{~d} x \mathrm{~d} t,
\end{aligned}
$$

for every $\varphi_{B} \in L^{2}\left(0, T ; H_{\text {null }}^{1}\left(\Omega^{B}\right)\right) \cap H_{0}^{1}\left(0, T ; L^{2}\left(\Omega^{B}\right)\right), \varphi_{D} \in L^{2}\left(0, T ; \mathcal{X}_{0}^{1}(\Omega)\right)$, where, as before, $\left[\varphi_{D}\right]=\varphi_{D}^{1}-\varphi_{D}^{2}$ and $\left[\varphi_{D}\right] \in H_{0}^{1}\left(0, T ; L^{2}(\Gamma)\right)$. Recalling (2.35), the thesis is achieved, up to an integration in time, once we have taken into account that 


$$
\int_{\Omega_{T}^{B}}\left(\sigma_{1}^{B}+\sigma_{2}^{B}\right) \nabla \widetilde{u} \cdot \nabla \varphi_{D}^{1} \mathrm{~d} x \mathrm{~d} t=\int_{\Omega_{T}^{B}}\left(f_{1}-f_{2}\right) \varphi_{D}^{1} \mathrm{~d} x \mathrm{~d} t,
$$

as follows from (3.1)-(3.3).

As a consequence of the previous results, we finally get our main theorem.

Theorem 3.6. Assume that $\sigma_{1}^{B}, \sigma_{2}^{B}, \sigma^{D}, \alpha, \beta, f_{1}, f_{2}, \bar{v}_{0}, s_{0}$, and $I_{\text {ion }}$ satisfy the assumptions stated in Sect. 2.3. Then, problem (2.26)-(2.34) admits a unique solution $(V, U) \in L^{2}\left(0, T ; H_{\text {null }}^{1}\left(\Omega^{B}\right)\right) \times L^{2}\left(0, T ; \mathcal{X}_{0}^{1}(\Omega)\right)$, such that $V \in \mathcal{C}^{0}\left([0, T] ; L^{2}\left(\Omega^{B}\right)\right)$ and $[U] \in \mathcal{C}^{0}\left([0, T] ; L^{2}(\Gamma)\right)$.

Proof. The proof can be obtained following the same approach as in [13] (see, also, [14, §2.4.1]). Indeed, recalling that the function $g$ appearing in the gating equation (2.12) is affine with respect to its second entry, problem (2.12)-(2.13) can be explicitly solved in term of $u_{1}^{B}-u_{2}^{B}=V$. Therefore, denoting by $\widetilde{w}_{V}$ such a solution and by $h(V):=I_{\text {ion }}\left(V, \widetilde{w}_{V}\right)$, we obtain that problem (2.26)-(2.34) is a nonlinear version of the problem considered in Proposition 3.5. Moreover, since the nonlinearity $h$ satisfies the assumptions [9, Definition 4.3.1], the thesis follows by the results in [9, Section 4.3] (see, also, [22, Ch. 6, Theorem 1.2]).

\section{Acknowledgements}

The first author is member of the Gruppo Nazionale per l'Analisi Matematica, la Probabilità e le loro Applicazioni (GNAMPA) of the Istituto Nazionale di Alta Matematica (INdAM). The second author is member of the Gruppo Nazionale per la Fisica Matematica (GNFM) of the Istituto Nazionale di Alta Matematica (INdAM). The last author wishes to thank Dipartimento di Scienze di Base e Applicate per l'Ingegneria for the warm hospitality and Università "La Sapienza" of Rome for the financial support.

Funding Open access funding provided by Universitá degli Studi di Roma La Sapienza within the CRUI-CARE Agreement.

Open Access. This article is licensed under a Creative Commons Attribution 4.0 International License, which permits use, sharing, adaptation, distribution and reproduction in any medium or format, as long as you give appropriate credit to the original author(s) and the source, provide a link to the Creative Commons licence, and indicate if changes were made. The images or other third party material in this article are included in the article's Creative Commons licence, unless indicated otherwise in a credit line to the material. If material is not included in the article's Creative Commons licence and your intended use is not permitted by statutory regulation or exceeds the permitted use, you will need to obtain permission directly from the copyright holder. To view a copy of this licence, visit http:// creativecommons.org/licenses/by/4.0/.

Publisher's Note Springer Nature remains neutral with regard to jurisdictional claims in published maps and institutional affiliations. 


\section{References}

[1] Amar, M., Andreucci, D., Bisegna, P., Gianni, R.: Existence and uniqueness for an elliptic problem with evolution arising in electrodynamics. Nonlinear Anal. Real World Appl. 6, 367-380 (2005)

[2] Amar, M., Andreucci, D., Timofte, C.: Homogenization of a modified bidomain model involving imperfect transmission. Commun. Pure Appl. Anal. 20(5), 1755-1782 (2021). https://doi.org/10.3934/cpaa.2021040

[3] Bendahmane, M., Karlsen, H.K.: Analysis of a class of degenerate reactiondiffusion systems and the bidomain model of cardiac tissue. Netw. Heterog. Media 1, 185-218 (2006)

[4] Boulakia, M.: Etude mathématique et numérique de modèles issus du domaine biomédical. Equations aux dérivées partielles [math.AP]. UPMC (2015)

[5] Boulakia, M., Cazeau, S., Fernández, M.A., Gerbeau, J.F., Zemzemi, N.: Mathematical modeling of electrocardiograms: a numerical study. Ann. Biomed. Eng. 38(3), 1071-1097 (2010)

[6] Boulakia, M., Fernández, M.A., Gerbeau, J.F., Zemzemi, N.: A coupled system of pdes and odes arising in electrocardiograms modelling. Appl. Math. Res. Express (2008). https://doi.org/10.1093/amrx/abn002

[7] Boulakia, M., Fernández, M.A., Gerbeau, J.F., Zemzemi, N.: Towards the numerical simulation of electrocardiograms. In: Sachse, F., Seemann, G. (eds.) Functional Imaging and Modeling of the Heart. FIMH 2007. Lecture Notes in Computer Science, vol. 4466, pp. 240-249. Springer, Berlin (2007)

[8] Bourgault, Y., Coudière, Y., Pierre, C.: Existence and uniqueness of the solution for the bidomain model used in cardiac electrophysiology. Nonlinear Anal. Real World Appl. 10(1) , 458-482 (2009)

[9] Cazenave, T., Haraux, A.: An Introduction to Semilinear Evolution Equations. Oxford Lecture Series in Mathematics and Its Applications, vol. 13. Oxford University Press, New York (1998)

[10] Clayton, R., Bernus, O., Cherry, E., Dierckx, H., Fenton, F., Mirabella, L., Panfilov, A., Sachse, F., Zhang, H.: Models of cardiac tissue electrophysiology: progress, challenges and open questions. Prog. Biophys. Mol. Biol. 104, 22-48 (2011)

[11] Collin, A., Imperiale, S.: Mathematical analysis and 2-scale convergence of an heterogeneous microscopic bidomain model. Math. Models Methods Appl. Sci. 28(5), 979-1035 (2018) 58

[12] Coudière, Y., Davidovic, A., Poignard, C.: The modified bidomain model with periodic diffusive inclusions. In: Murray, A. (ed.) Computing in Cardiology Conference (CinC), pp. 1033-1036. IEEE (2014). https://ieeexplore.ieee.org/ abstract/document/7043222

[13] Coudière, Y., Davidovic, A., Poignard, C.: Modified bidomain model with passive periodic heterogeneities. In: DCDS, Series S (2019). https://doi.org/10. 3934/dcdss.2020126

[14] Davidovic̀, A.: Multiscale mathematical modelling of structural heterogeneities in cardiac electrophysiology. General Mathematics [math.GM]. Université de Bordeaux, NNT:2016BORD0448 (2016)

[15] Grandelius, E., Karlsen, K.H.: The cardiac bidomain model and homogenization. Netw. Heterog. Media 14(1), 173-204 (2019) 
[16] Jerez-Hanckes, C., Pettersson, I., Rybalko, V.: Derivation of cable equation by multiscale analysis for a model of myelinated axons. DCDS Ser. B 25(3), 815-839 (2020)

[17] Kajiwara, N.: On the bidomain equations as parabolic evolution equations (2020) (preprint)

[18] Kavian, O., Leguèbe, M., Poignard, C., Weynans, L.: Classical electropermeabilization modeling at the cell scale. J. Math. Biol. 68, 235-265 (2014)

[19] Keener, J., Sneyd, J.: Mathematical Physiology. Springer, New York (2004)

[20] Krassowska, W., Neu, J.: Homogenization of syncytial tissues. Crit. Rev. Biomed. Eng. 21, 137-199 (1992)

[21] Nagumo, J., Arimoto, S., Yoshizawa, S.: An active pulse transmission line simulating nerve axon. Proc. Inst. Radio Eng. 50, 2061-2070 (1962)

[22] Pazy, A.: Semigroups of Linear Operators and Applications to Partial Differential Equations, vol. 44. Springer Science \& Business Media, Berlin (2012)

[23] Pennacchio, M., Savaré, G., Franzone, P.C.: Multiscale modeling for the bioelectric activity of the heart. SIAM J. Math. Anal. 37(4), 1333-1370 (2005)

[24] Veneroni, M.: Reaction-diffusion systems for the macroscopic bidomain model of the cardiac electric field. Nonlinear Anal. Real World Appl. 10, 849-868 (2009)

[25] Zeidler, E.: Nonlinear Functional Analysis and Its Applications, vol. II/A. Springer, Berlin (1990)

[26] Zemzemi, N.: Theoretical and numerical study of the electric activity of the heart. Modeling and numerical simulation of electrocardiograms. Mathematics [math]. Université Paris Sud-Paris XI (2009) (English)

\section{Amar and D. Andreucci}

Dipartimento di Scienze di Base e Applicate per l'Ingegneria

Sapienza-Università di Roma

Via A. Scarpa 16

00161 Rome

Italy

e-mail: micol.amar@uniroma1.it

C. Timofte

Faculty of Physics

University of Bucharest

P.O. Box MG-11, Bucharest-Magurele

Romania

Received: June 23, 2020.

Revised: December 16, 2020.

Accepted: August 3, 2021. 